\title{
Identifying Key Factors Affecting Transnational Knowledge Transfer
}

\author{
Yanqing Duan ${ }^{1}$ and Wanyan Nie \\ University of Bedfordshire Business School \\ Elayne Coakes \\ Westminster Business School \\ University of Westminster
}

We wished to further understand Transnational Knowledge Transfer (TKT) in a not-for-profit context. To accomplish this, we explored, verified, and mapped out the key factors affecting TKT using a four-component framework in the context of publically funded knowledge transfer (KT) projects. The Delphi technique was used to explore, identify, and verify the relevant key factors; twenty-four major factors were identified in the first round and more than half of the experts agreed on the top ten key factors in the second round. In addition, a number of new factors were identified and some findings that contradicted prior research were revealed. Our findings can help practitioners develop a more focused approach in dealing with the most significant factors (or bottlenecks) in KT.

\footnotetext{
${ }^{1}$ Corresponding author - Prof Yanqing Duan, University of Bedfordshire Business School, Luton LU1 3JU, UK, Tel: +44 (0) 1582743134' Fax: +44 (0) 01582 743172, email: yanqing.duan@ beds.ac.uk
} 


\section{Identifying Key Factors Affecting Transnational Knowledge Transfer}

\section{Introduction}

Today, knowledge is shared between geographically dispersed individuals and organisations, and across cultural and national boundaries. Therefore, understanding how knowledge can be transferred from one organisation or sub-unit to another is extremely important, Knowledge Transfer (KT) is one of the most important stages in knowledge management, but is considered to be laborious and time consuming to achieve effectively.

We define transnational knowledge transfer (TKT) as KT across different countries, or acrossborders. It happens through Multi-National Corporations (MNC), Transnational organisations [1], International Joint Ventures (IJV), and international projects supported by governments, the United Nations, the European Commission, and national and international funding organisations helping developing countries.

While all knowledge transfer faces some obstacles, cross-border KT faces extra challenges due to cross-cultural, political, economic, and geographical gaps. Knowledge sharing is often seen as resulting in a loss of power, and, as a result, knowledge that should be transferred is often withheld, leading to inefficiency. Furthermore, theories of KT at the local, individual, intra- or inter-organisational level cannot be directly applied to global TKTs.

There are a number of gaps in our understanding of transnational knowledge transfer. Firstly, the literature on it mostly examines business related knowledge transfer; e.g., [2-4] and little attention was paid to transnational knowledge transfer activities at the inter-organisational level [5]. Secondly, we found no work that attempted to examine transnational knowledge transfer in the context of publically funded projects. Thirdly, though there are many factors that affect 
knowledge transfer, no research has been performed to either confirm their relevance, or explore new or context specific factors.

We sought to fill these gaps by asking: "What are the most salient factors that affect transnational knowledge transfer success in the context of publically funded projects?"

Data for our research was collected using a Delphi study with a panel of experts involved in the European Commission's (EC) Europe Aid Asia Programme (EAAP). This Programme promotes and funds cross-country and cross-continent KT projects. It has different sub-programmes, such as Asia Invest, Asia Pro Eco, Asia IT\&C, and Asia-Link, which explicitly focus on improving knowledge cross-flow and the quality of Euro-Asia partnerships. However, each sub-programme only focused on one particular application area of collaboration and knowledge transfer: a brief description of these sub-programmes is provided in Appendix 2. By 2007, EAAP had funded about $800 \mathrm{KT}$ projects supporting the exchange of methods and best practices among European and Asian higher education institutions, companies, and the public sector. This international and regional integration and co-operation enabled countries to address cross-border challenges, such as environment, health, and economic co-operation; it also offered a platform to facilitate knowledge sharing and transfer between developed and developing countries. Knowledge was transferred via channels and activities such as training and education, business networking, development of new business opportunities, exchange of ideas, and collaboration in research and development.

\section{Literature Review}

\subsection{Our definition of knowledge transfer}

Knowledge transfer has been defined as a process by which:

- one organizational unit (e.g. a group, department, or division) passes its experience to another [6]; 
- $\quad$ systematically organized information and skills are exchanged between entities [7];

- knowledge is exchanged between or among individuals, teams, groups, or organisations.

Some authors use the terms 'knowledge sharing' or "organisational learning" as synonyms of "knowledge transfer".

From an organisational perspective, TKT can occur at both individual and organisational levels, within a community or partnership; and inter-organisationally among organisations in different nations - when sponsored by third parties such as international or national charities or governing bodies. Transnational organisations focus on a network of competences, which may include several organisations. In a transnational context, knowledge workers may be dispersed through both virtual working practices and throughout the organisation.

\subsection{Knowledge transfer levels}

At the basic level, knowledge is possessed, created, shared, and leveraged by individuals without individuals' involvement, knowledge cannot be transferred.

Knowledge transfer at the intra and trans-national organisational level views an organisation as an entity within which knowledge is created and shared. Thus organizational practices, structure, and processes play a critical role in articulating and amplifying knowledge developed by individuals.

When organisations engage in inter-organisational knowledge transfer, they open their borders to flows of information and knowledge from external networks; sophisticated knowledge codification techniques allow knowledge sharing among organisations, even competitors strategic alliances, multinational and transnational enterprises, and international projects. TKT has a challenge: its participants are often separated by time, space, culture and language [8], which may limit their ability to access, share, and absorb knowledge effectively. 


\subsection{Knowledge transfer models}

There has been a surge of interests in KT in recent years. A significant number of papers have discussed attempts to develop knowledge transfer models and frameworks to show the transfer process, the key actors, the transfer channels, and influential factors, for example, to knowledge at the individual level [9]; at the intra-organisational level [10], and inter-organisationally [11], and at the transnational level [12].

\section{$\underline{\text { Individual knowledge transfer models }}$}

Here, knowledge is embodied in people and created and developed by individuals. Sharing individual knowledge is essential for the creation, dissemination, and management of knowledge at all higher levels and cannot be transferred without involvement of the individual, which requires learning, and Ipe's conceptual framework of knowledge sharing which involved four influential factors (the nature of knowledge, opportunity and motivations to share, and the culture of the work environment) and their relationships.

\section{$\underline{\text { Intra-organisational knowledge transfer models }}$}

Several models and frameworks have been developed to understand knowledge transfer phenomena in an intra-organizational context. Szulanski's model [13] had four stages: Initiation of a transfer; initial implementation; ramp-up to satisfactory performance; and integration, which requires follow-through and evaluation in order to integrate or transform the new practices into the prior practices of the KT recipient. Goh's [14] integrative framework has been cited as the model that integrates key factors that have significant influence on effective transfer of knowledge; this assumes that knowledge transfer requires a co-operative and collaborative culture. Orendorff et al. emphasised the difference between knowledge transfer and knowledge translation, and proposed an intra-organisational transfer framework that was a knowledge utilisation model. The framework applies to both horizontal KT (involving knowledge already in 
use in a similar organisation) and vertical KT (knowledge put to use for the first time, transferred from a dissimilar unit).

$\underline{\text { Inter-organisational knowledge transfer models }}$

When transferring knowledge between organisations, the interfacing problem has to be considered; it has led to several other models (e.g., [15-17]. Building on previous work on interorganisational knowledge transfer, Abou-Zeid conceptualised inter-organisational knowledge transfer as a culturally aware four-stage process involving initialisation, inter-relation, implementation, and internalisation. This allowed a close examination of how the culture traits of source and recipient firms at different levels affected each stage of the process. KT process and factors were also addressed by Cranefield and Yoong ], and. in SMEs by Chen et al. [11] who assumed that transfer followed a five stage process: identification; negotiation; selection; interaction; and conversion.

\subsection{Knowledge transfer models at the transnational level}

Research appears to have been limited to the context of MNC and IJV. Schlegelmilch and Chini in proposing a conceptual framework for effective marketing addressed the impact of organisational distance, cultural distance, strategic mandate, and ability to engage in knowledge transfer; Cummings and Teng integrated inter- and intra- organisational knowledge transfer. A model developed by Miesing et al. indicated that effective intra-organisational knowledge transfer across nations requires the creation of social capital between members and requires a collective transnational strategy.

These models offered a dynamic view of knowledge transfer and depicted the causal relationship between transfer success and the influential factors, however, it should be noted that most of them were not empirically tested or validated. 


\subsection{Factors affecting knowledge transfer}

We decided that the model suggested by Albino et al. [18] had a comprehensive coverage of key elements and their relationships was suitable for our data analysis. A summary of the factors is presented in Table 1.

(Table 1. A summary of the literature on factors affecting knowledge transfer is about here)

However, there seemed to be a need to develop a knowledge transfer taxonomy for better understanding of the complex issues. Table 2 shows our attempt to present a preliminary framework for developing a comprehensive taxonomy.

(Table 2. A taxonomy for understanding knowledge transfer is about here)

Our research was therefore an attempt to identify and confirm the key factors in the domain of not-for-profit organizations.

\section{Research Method}

\subsection{The Delphi method}

The Delphi method seeks to obtain consensus on the opinions of experts through a series of questionnaires that collect and aggregate informed judgements on specific questions or issues.

\subsection{Identification of experts}

A Delphi study [19] requires qualified experts with a deep understanding of the issues,. All experts in our study were people who had been actively involved in a number of EU funded knowledge transfer projects as manager or principal investigator, or had been involved in TKT projects and understood knowledge transfer based on their publications. The set of criteria for identifying of relevant experts is shown in Table 3. 
(Table 3. A summary of the expert selection process is about here)

Low responsibility or involvement in only one project, caused a potential expert's elimination.

\subsection{Delphi survey administration}

The first round of the Delphi survey was qualitative the experts were sent, via email, a cover letter summarizing factors considered important in the current literature with a link to an openended questionnaire (an MSWord file). The experts were asked to list their five most important factors and give explanations why they were considered important. Twenty out of the sixty experts responded after follow up, an effective response rate of $33 \%$.

Content analysis was used to analyse the panel's feedback and group the factors into themes. Analysis reliability was achieved through independent categorisation (inter-rating) by two researchers, followed by several rounds of modifications by the authors of this paper and independent researchers.

The factors that experts identified and the justifications they provided were not always consistent, therefore, their explanations were used to change categorisation of the factors.

In the second round, the number of instances that fell into each category and the descending order frequencies of factors were sent back to the experts, who were also sent their previous answers. Seventeen experts responded to the second round an $85 \%$ response rate.

When considering the level of consensus to be required, two or three rounds are normally preferred. The controlled feedback from round 2 in our Delphi study resulted in ten factors receiving between $53 \%$ and $88 \%$ agreement and therefore, we believed that a further round of was unnecessary. 


\section{Survey Results}

\subsection{Participants'profile}

Table 4 gives the demographics of participating experts. Most of the partners in EU funded projects are academic institutions, therefore it is not surprising that most survey participants (82\%) were academics; they could also be classified as practitioners of KT as this had been part of their roles in their EU project.

(Table 4. Profile of participating experts is about here)

\subsection{Participants' contributions}

The nature of the open and non-prescriptive questions in the first round offered freedom for experts to contribute effectively. The qualitative data provided a rich and diverse content, which, by its own nature, added value to our study.

Content analysis of the first round generated twenty-four categorised factors underpinned by many differing explanations. These twenty four factors were sent to the responding experts in the second round to re-collect a consensus view on the most important factors. The initial levels of agreement with themes were sent to the list of experts and they were asked to review and revise their responses. .Table 5 summarizes the two rounds.

(Table 5. Information on factors identified in rounds $1 \& 2$ is about here) 
5. Discussion I put the subsections of this section into a new appendix -- 2- they are too detailed for the main body of the paper

Ten factors were selected by more than $50 \%$ of the experts as the key factors affecting TKT projects. Relationship and cultural awareness were regarded by a majority of our experts (88\% and $82 \%$ ), as the key factors affecting KT success, followed by common language (76\% plus). Other factors, such as motivation, knowledge distance, selection of appropriate partners, objectives and focus, transfer channel, trust, and openness were also considered important by more than $50 \%$ of the experts. The consensus rating of some factors dramatically increased in the second round. These are discussed in detail in Appendix 2.

\section{Implications}

Globalisation has pushed organisations to search for new opportunities, extend existing business boundaries, and develop international collaboration. TKT in the public funded sector, is more complicated and difficult to achieve than KT at individual or organisational leveld,. Therefore, a better understanding of enhancing the effectiveness of transnational knowledge transfer is necessary.

To analyse the findings and better understand the research implications, all factors identified by experts this were mapped onto the Albino et al [18] four-component model in Figure 2. zzz

Figure 2. A framework for analysing factors affecting transnational knowledge transfer is about here.

Five of the ten key factors - culture awareness, motivation, knowledge distance, trust and openness - are related to the KT actors. This has significant implications on TKT success from both theoretical and practical perspectives, as actors in KT can be drivers or barriers to successful transfer. The challenge is in influencing and managing the actors to provide positive TKT impact. 
Organisations need to offer motivational incentives to staff. Also, as the knowledge gap between senders and receivers may negatively affect transfer success, organisations must decide if the receivers are capable of receiving, absorbing, and re-using the knowledge transferred - in the manner in which it is transferred. If the knowledge distance is high, a knowledge intermediary might be required.

It is also noted that the relationship between and selection of, appropriate partners are important factors. In the KT process, knowledge cannot flow by itself; human subjects acting within a contextual environment enable the transfer. Relationship as a contextual factor was seen, by the experts, as essential for TKT success. The realisation of KT depends not only on the people who interpret, organise, plan, develop and execute, and use this knowledge, but also on its specific situation and context. Selection of appropriate partners for KT projects was seen by the experts as a key factor in transfer.

Language and the Transfer Channel are key factors of the transfer media: "the selection of the appropriate communication channels and mechanisms is of vital importance for the success of the KT task" (Expert 6). Actors should make sure that the partners agree and implement effective transfer channels.

In terms of KT content, objectives and focus were found to be key factors, demonstrating that organisations should have clear objectives and should focus on what, and how, knowledge can be transferred.

Although trust has been widely acknowledged in the literature as an important factor in knowledge sharing and transfer, it was seen as less significant in transnational KT. The experts rated it below all other key factors.

\section{Conclusions}


Our research empirically explored and verified the key factors affecting TKT success. We used a Delphi method, to analyse and map out these factors using a four-component framework. The data was collected from a panel of experts who had extensive knowledge and experience in TKT through the EAAP. Twenty experts participated. Ten factors were highly rated: Relationship, Culture awareness, Language, Motivation, Knowledge distance, Objectives and focus, Transfer channel, Selection of appropriate partners, Trust, and Openness. The expert panel identified a number of factors not formally recognised in prior research, including: objectives and focus; selection of appropriate partners; respect; use of expert trainers; project flexibility; institutional collaboration; expert subject knowledge; policy framework/bureaucratic procedures; and topic timeliness.

Our findings can assist practitioners in developing a focused approach to factors (including bottlenecks) that affect successful KT.

Our research had some limitations. The Delphi method has many merits, but also has weaknesses. Conducting a Delphi survey is time-consuming, and often precludes statistical analysis. Relies on the expert panel to arrive at a consensus; however, the panel may be biased.

Finally, we strongly believe that the key factors unveiled here are applicable to any types of TKT, but caution is needed to avoid overlooking other context-specific factors for commercial knowledge transfer.

\section{Acknowledgement}

Authors are very grateful for the contributions made by twenty experts. It would not be possible to complete this research without their collaboration. Their time, effort, knowledge, and opinions are greatly appreciated.

\section{References}


1. Coakes, E., Storing and sharing knowledge - supporting the management of knowledge made explicit. The Learning Organization 2008. 13(6): p. 579-593.

2. Gooderham, P.N., Enhancing knowledge transfer in multinational corporations: a dynamic capabilities driven model. Management Research \& Practice, 2007. 5: p. 34-43.

3. Gupta, A.K. and V. Govindarajan, Knowledge flows within multinational corporations. Strategic Management Journal, 2000. 21(4): p. 473-496.

4. Shachaf, P., Cultural diversity and information and communication technology impacts on global virtual teams: An exploratory study. Information \& Management, 2008. 45(2): p. 131-142.

5. Chevrier, S., Cross-cultural management in multinational project groups. Journal of World Business, 2003. 38(2): p. 141-149.

6. Argote, L. and P. Ingram, Knowledge transfer: A basis for competitive advantage in firms. Organizational Behavior and Human Decision Processes, 2000. 82(1): p. 150-169.

7. Wong, Y.Y., et al., Organisational Learning and the Risks of Technology Transfers in China. Management Research News, 2003. 26(12): p. 1-11.

8. Schlegelmilch, B.B. and T.C. Chini, Knowledge transfer between marketing functions in multinational companies: a conceptual model. International Business Review, 2003. 12(2): p. 215-232.

9. Ipe, M., Knowledge Sharing in Organizations: A Conceptual Framework. Human Resource Development Review, 2003. 2(4): p. 337-359.

10. Miesing, P., M.P. Kriger, and N. Slough, Towards a model of effective knowledge transfer within transnationals: The case of Chinese foreign invested enterprises. Journal of Technology Transfer, 2007. 32(1-2): p. 109-122.

11. Chen, S., et al., Toward understanding inter-organizational knowledge transfer needs in SMEs: insight from a UK investigation. Journal of Knowledge Management, 2006. 10(3): p. 6-23.

12. Duan, Y., X. Xu, and Z. Fu, Understanding Transnational Knowledge Transfer, in The 7th European Conference on Knowledge ManagementSept 2006, Academic Conference Limited: Budapest, Hungary.

13. Szulanski, G., The process of knowledge transfer: A diachronic analysis of stickiness. Organizational Behavior and Human Decision Processes, 2000. 82(1): p. 9-27.

14. Goh, S.C., Managing effective knowledge transfer: An integrative framework and some practice implications. Journal of Knowledge Management, 2002. 6(1): p. 23-30.

15. Abou-Zeid, E.-S., A culturally aware model of inter-organizational knowledge transfer. Knowledge Management Research and Practice 2005. 3(3): p. 146-155.

16. Agrawal, A., University-to-industry knowledge transfer: literature review and unanswered questions. International Journal of Management Reviews, 2001. 3(4): p. 285302.

17. Mohr, J.J. and S. Sengupta, Managing the paradox of inter-firm learning: The role of governance mechanisms. The Journal of Business \& Industrial Marketing, 2002. 17(4): p. 282-301.

18. Albino, V., A.C. Garavelli, and G. Schiuma, Knowledge transfer and inter-firm relationships in industrial districts: the role of the leader firm. Technovation, 1998. 19(1): p. 53-63.

19. Okoli, C. and S.D. Pawlowski, The Delphi method as a research tool: an example, design consideration and applications. Information and Management, 2004. 42(1): p. 15-29.

20. Cummings, J.L. and B.S. Teng, Transferring R\&D knowledge: the key factors affecting knowledge transfer success. Journal of Engineering and Technology Management, 2003. 20(1-2): p. 39-68. 
21. Minbaeva, D., et al., MNC knowledge transfer, subsidiary absorptive capacity, and HRM. Journal of International Business Studies, 2003. 34(6): p. 586-599.

22. Osterloh, M. and B.S. Frey, Motivation, knowledge transfer, and organizational forms. Organization Science, 2000. 11(5): p. 538-550.

23. Kayes, D.C., B. Kayes Anna, and Y. Yamazaki, Essential competencies for cross-cultural knowledge absorption. Journal of Managerial Psychology, 2005. 20(7): p. 578-589.

24. Delios, A. and I. Bjorkman, Expatriate Staffing in Foreign Subsidiaries of Japanese Multinational Corporations in the PRCc and the United States. International Journal of Human Resource Management, 2000. 11(2): p. 278-293.

25. De Long, D.W. and L. Fahey, Diagnosing cultural barriers to knowledge management. Academy of Management Executive, 2000. 14(4): p. 113-127.

26. Inkpen, A.C. and W. Pien, An examination of collaboration and knowledge transfer: China-Singapore Suzhou Industrial Park. Journal of Management Studies, 2006. 43(4): p. 779-811.

27. Seibert, S.E., M.L. Kraimer, and R.C. Liden, A social capital theory of career success. Academy of Management Journal, 2001. 44(2): p. 219-237.

28. Hansen, M.T. and B. Løvås, Leveraging Technological Competencies. Strategic Management Journal, 2004. 25(8-9): p. 801-822.

29. Pedersen, T., B. Petersen, and D. Sharma, Knowledge Transfer Performance of Multinational Companies. Management International Review, 2003. 43(3): p. 69-90.

30. Syed-Ikhsan, S.O.S. and F. Rowland, Knowledge management in a public organization: a study on the relationship between organizational elements and the performance of knowledge transfer. Journal of Knowledge Management, 2004. 8(2): p. 95-111.

31. Chow, C.W., F.J. Deng, and J.L. Ho, The Openness of Knowledge Sharing within Organizations: A Comparative Study of the United States and the People's Republic of China. Journal of Management Accounting Research, 2000. 12(1): p. 65-95. 
Table 1. A summary of the literature on factors affecting knowledge transfer

\begin{tabular}{|c|c|c|}
\hline Knowledge transfer component & Associated factor & Recent references \\
\hline \multirow{7}{*}{$\begin{array}{l}\text { Actors: } \\
\text { involved in the knowledge transfer process. } \\
\text { always central to the process of. Three key actors are } \\
\text { generally identified: sender; recipient; and } \\
\text { intermediary. }\end{array}$} & Openness & {$[14]$} \\
\hline & Prior Experience and Knowledge & [20]; [3] \\
\hline & Trust & {$[3]$} \\
\hline & Motivation & [21]; [22]; [13] \\
\hline & Leadership & {$[14]$} \\
\hline & Absorptive capacity & {$[23]$} \\
\hline & Competence & {$[24]$} \\
\hline \multirow{5}{*}{$\begin{array}{l}\text { Context: } \\
\text { Where the interaction takes place. } \\
\text { Transferring knowledge is contextually bound, so its } \\
\text { transfer is constrained by the contexts in which it is } \\
\text { embedded. }\end{array}$} & Culture difference & $\begin{array}{l}{[15] ;[25] ;[14] ;[9] ;} \\
{[8]}\end{array}$ \\
\hline & Relationship & {$[26]$} \\
\hline & Social capital & [27]; \\
\hline & Physical distance & {$[28]$} \\
\hline & Organizational infrastructure & {$[14]$} \\
\hline $\begin{array}{l}\text { Content: } \\
\text { To be transferred between actors. }\end{array}$ & Nature and content of knowledge & {$[14],[13]$} \\
\hline \multirow{4}{*}{$\begin{array}{l}\text { Media: } \\
\text { By which the transfer is carried out. }\end{array}$} & Language and translation & {$[23]$} \\
\hline & Communication channels & \begin{tabular}{|l|l}
{$[29]$} \\
\end{tabular} \\
\hline & Transfer channel & {$[29]$} \\
\hline & Use of ICT & {$[30]$} \\
\hline
\end{tabular}


Table 2. A taxonomy for understanding knowledge transfer

\begin{tabular}{|c|c|c|}
\hline Attribute & \multicolumn{2}{|c|}{ Example } \\
\hline Knowledge transfer Level & \multicolumn{2}{|c|}{$\begin{array}{ll}\text { - } & \text { Individual level } \\
\text { - } & \text { Intra-organisational level } \\
\text { - Inter-organisational level } \\
\text { - } & \text { Transnational level } \\
\end{array}$} \\
\hline Knowledge transfer model & $\begin{array}{ll}\text { - } & \text { Process based model } \\
\text { - } & \text { Factor based model } \\
\text { - } & \text { Process and factor } \\
\text { model } \\
\text { - } \\
\text { Component based model }\end{array}$ & $\begin{array}{ll}\text { - } & \text { Individual KT model } \\
\text { - } & \text { Intra-organisational KT model } \\
\text { - } & \text { Inter-organisational KT model } \\
\text { - } & \text { Transnational KT model }\end{array}$ \\
\hline $\begin{array}{l}\text { Knowledge transfer } \\
\text { component }\end{array}$ & \multicolumn{2}{|l|}{$\begin{array}{ll}\text { From }[18]: \\
\text { - } & \text { Actors } \\
\text { - } & \text { Content } \\
\text { - } & \text { Context } \\
\text { - } & \text { Media }\end{array}$} \\
\hline Knowledge transfer factor & \multicolumn{2}{|l|}{$\begin{array}{l}\text { For example (see table } 1 \text { ): } \\
\text { - } \quad \text { Actor related factors } \\
\text { - } \quad \text { Content related factors } \\
\text { - } \quad \text { Context related factors } \\
\text { - } \quad \text { Media related factors } \\
\end{array}$} \\
\hline
\end{tabular}


Table 3. The expert selection process

Expert selection process

1. Development of the expert selection criteria

\section{Outcome}

An expert should meet one of the following criteria:

- Manager or practitioner who was directly involved in project management;

- Senior academis who had participated in projects and demonstrated sound understanding of KT through relevant publications;

- $\quad$ Principal investigators involved in more than one project.

2. Collection of information on The published EAAP directory was analysed. A total of $\mathbf{7 1 8}$ projects with a the EU Asia Aid projects $\quad$ valid website were found.

3. Identification and evaluation Each web site and its current status were examined. Those projects considered to of relevant projects be focusing on TKT activities, having a good quality web site and still current or had completed recently were selected. 151 projects were chosen for further examination.

4. Identification of the An expert was identified from each project. potential expert in each project

5. Final selection of the We visited the expert's personal web sites and publication record in relevant experts areas. At the end of this process, $\mathbf{6 0}$ experts were selected for our Delphi survey. 
Table 4. Profile of participating experts

\begin{tabular}{|l|c|c|c|c||}
\hline & \multicolumn{2}{|c|}{$\mathbf{1}^{\text {st }}$ round } & \multicolumn{2}{c||}{$\mathbf{2}^{\text {nd }}$ round } \\
\hline & Number (n=20) & $\begin{array}{c}\text { \% of } \\
\text { participants }\end{array}$ & $\begin{array}{c}\text { Number } \\
\text { (n=17) }\end{array}$ & $\begin{array}{c}\text { of } \\
\text { participants }\end{array}$ \\
\hline Job Title & & & & \\
\hline Professor & 5 & $25 \%$ & 5 & $30 \%$ \\
\hline Project manager & 9 & $45 \%$ & 8 & $47 \%$ \\
\hline Senior manager (Director) & 6 & $30 \%$ & 4 & $23 \%$ \\
\hline Type of Organization & & & & \\
\hline Academic Institution & 16 & $80 \%$ & 14 & $82 \%$ \\
\hline Public Organization & 2 & $10 \%$ & 1 & $6 \%$ \\
\hline Commercial Organization & 2 & $10 \%$ & 2 & $12 \%$ \\
\hline No. of EU/C Projects involved & & & & \\
\hline$\geq 3$ & 13 & $65 \%$ & 10 & $59 \%$ \\
\hline $1-2$ & 7 & $35 \%$ & 7 & $41 \%$ \\
\hline Years of experience in EU/C Projects & & & & \\
\hline$\geq 10$ & 4 & $20 \%$ & 4 & $23 \%$ \\
\hline $4-9$ & 11 & $55 \%$ & 8 & $47 \%$ \\
\hline$\leq 3$ & 5 & $25 \%$ & 5 & $30 \%$ \\
\hline Type of EU/C Programme involved in & & & & \\
\hline Asia-Link & 12 & $60 \%$ & 10 & $59 \%$ \\
\hline Asia Pro eco & 8 & $40 \%$ & 5 & $30 \%$ \\
\hline Asia ICT & 6 & $30 \%$ & 5 & $50 \%$ \\
\hline Asia Invest & 2 & $10 \%$ & 2 & $12 \%$ \\
\hline Others & $40 \%$ & 5 & $30 \%$ \\
\hline
\end{tabular}


Table 5. Information on factors identified in rounds $1 \& 2$

\begin{tabular}{|c|c|c|c|c|c|}
\hline \multirow{2}{*}{ Factors } & \multicolumn{2}{|c|}{ Round 1} & \multicolumn{2}{|c|}{ Round 2} & \multirow{2}{*}{ Change } \\
\hline & $\mathbf{N}=\mathbf{2 0}$ & $\%$ & $\mathbf{N}=\mathbf{1 7}$ & $\%$ & \\
\hline Relationship & 13 & $65 \%$ & 15 & $88 \%$ & $+23 \%$ \\
\hline Culture awareness & 8 & $40 \%$ & 14 & $82 \%$ & $+42 \%$ \\
\hline Language & 8 & $40 \%$ & 13 & $76 \%$ & $+36 \%$ \\
\hline Motivation & 8 & $40 \%$ & 11 & $65 \%$ & $+25 \%$ \\
\hline Knowledge distance & 10 & $50 \%$ & 11 & $65 \%$ & $+15 \%$ \\
\hline Objectives and focus & 7 & $35 \%$ & 10 & $\mathbf{5 9 \%}$ & $+24 \%$ \\
\hline Transfer channel & 5 & $25 \%$ & 10 & $59 \%$ & $+34 \%$ \\
\hline Selection of appropriate partners & 1 & $5 \%$ & 9 & $53 \%$ & $+48 \%$ \\
\hline Trust & 7 & $35 \%$ & 9 & $53 \%$ & $+18 \%$ \\
\hline Openness & 6 & $30 \%$ & 9 & $53 \%$ & $+23 \%$ \\
\hline Use of ICT & 3 & $15 \%$ & 6 & $35 \%$ & $+20 \%$ \\
\hline Respect & 4 & $20 \%$ & 6 & $35 \%$ & $+15 \%$ \\
\hline Knowledge absorption & 3 & $15 \%$ & 6 & $35 \%$ & $+20 \%$ \\
\hline Use of expert trainers & 1 & $5 \%$ & 5 & $29 \%$ & $+24 \%$ \\
\hline Flexibility & 1 & $5 \%$ & 5 & $29 \%$ & $+24 \%$ \\
\hline Instrumental content & 3 & $15 \%$ & 5 & $29 \%$ & $+14 \%$ \\
\hline $\begin{array}{l}\text { Nature of knowledge } \\
\text { to be transferred }\end{array}$ & 3 & $15 \%$ & 4 & $24 \%$ & $+9 \%$ \\
\hline Partners' existing networks & 1 & $5 \%$ & 4 & $24 \%$ & $+19 \%$ \\
\hline Institutional collaboration & 1 & $5 \%$ & 4 & $24 \%$ & $+19 \%$ \\
\hline Causal ambiguity & 1 & $5 \%$ & 3 & $18 \%$ & $+13 \%$ \\
\hline $\begin{array}{l}\text { Expertise knowledge } \\
\text { on the subject }\end{array}$ & 1 & $5 \%$ & 3 & $18 \%$ & $+13 \%$ \\
\hline $\begin{array}{l}\text { Policy framework/ } \\
\text { Bureaucratic procedures }\end{array}$ & 2 & $10 \%$ & 2 & $12 \%$ & $+2 \%$ \\
\hline Timeliness of the topics & 1 & $5 \%$ & 2 & $12 \%$ & $+7 \%$ \\
\hline
\end{tabular}


Figure 2. A framework for analysing factors affecting transnational knowledge transfer

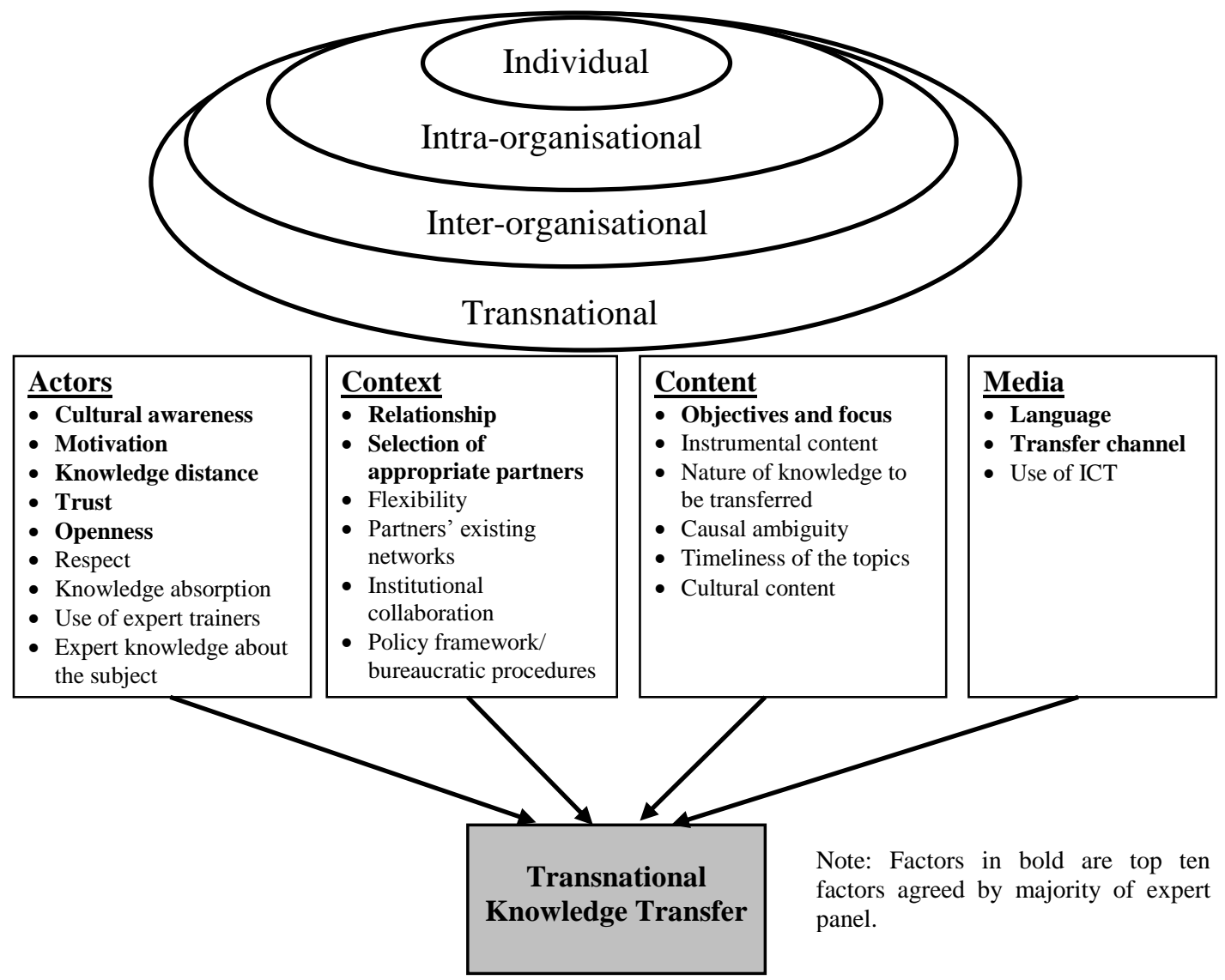




\section{Appendix 1 - Expert Comments during the second round of the Delphi Study}

\section{Relationship}

Almost all the experts agreed that relationship plays a critical role in TKT success. As Chevrier explained, when people are well acquainted with each other, they set up working arrangements more easily. "A good relationship is arguably the most important factor to make an international project work successfully. Without such a relationship, the project is likely to be almost useless." (Expert 11). “... this is more evident when working with Asian partners.” (Expert 2) because "Personal contacts and friendship is the basis of any fruitful collaboration; in particular if scientific knowledge transfer or sharing of ideas is involved” (Expert 16).

One social phenomenon, Guanxi, is particular to Chinese life, and prevalent throughout South East Asia. It involved a relationship between organisations or persons, not only to family and friends but also to social connections based on mutual interest and benefit. Such a reciprocal relationship helps establish a harmonious environment, facilitating KT across cultures, and thus, can be an important facet of TKT. Success of transnational organisations is dependent on people from different cultures, and relationships. "Participants will overcome practical differences to support partners because of relationship, not the legal contract". (Expert 4); "The setting up of a friendly relationship helps to bridge the communication differences and enables all to openly discuss differences in knowledge approaches and eases transnational knowledge transfer". (Expert 10)

\section{Cultural Awareness}

Culture is a key dimension of KT and most experts supported this saying: "A substantial difference is observed in the way to organise terms and work with Asian partners" (Expert 6); "Culture understanding enables effective communication" (Expert 3). The reasons given to justify these statements included: “Awareness of others' culture facilitates positive dialogue and 
interest in others' cultural background and stimulate enthusiasm". (Expert 11); and "It is important that all partners do know the respective cultural backgrounds" (Expert 15).

In the EAAP cross-cultural teaching and learning was seen as a major KT channel. Experts pointed out that:"teachers from the West should be aware of cultural misunderstanding of learning, be aware of the different teaching norms in their recipient countries" (Expert 13); and "Consider pedagogical culture-differences in understanding the nature of teaching and learning" (Expert 7).

\section{Language}

Language is also one of the major issues, as it is not only related to the differences in natural languages, but also the different ways in which people use the same language. In our survey, the agreement on language as a key factor changed from $40 \%$ in the first round to $76 \%$ in the second. Experts indicated that language could be "a hamper" (Expert 14); "a major barrier" (Expert 2); "an added problem" (Expert 8); and "an additional resistance" (Expert 10) to KT successs. "Clarity of language is vital, [partners should] agree [with] what terms mean to each partner" (Expert 4).

Although English is the predominant language of the EAAP, partners from Asian countries did not seem to have a good command of English. This could cause communication problems, especially for virtual communications, and undermine their ability to share or transfer knowledge. When advanced knowledge is transferred from developed to developing countries, knowledge needs to be codified and translated to create a common cognitive background. As the translator has a preferred language, the knowledge conversion process is not culturally neutral,. One expert noted that "the quality of translation" (Expert 18) is a factor affecting TKT. Three issues were highlighted in the quality of translation - "ambiguity, interference and lack of equivalence" 
(Expert 18), and, in order to improve the situation, one expert suggested "patience is needed when having a discussion with people not using their first language”, (Expert 11).

\section{Motivation}

Motivation as a key factor [21, 22] was extensively echoed by the experts, emphasising that it leads to KT success: "The need of a specific knowledge to solve a problem and/or to be promoted within the institution and/or to be able to apply for other national projects" (Expert 10); "a need or desire to seek or accept knowledge from outside" (Expert 18); and "it is prerequisite to have a common reason for collaboration”. (Expert 12). Therefore, “All partners should be aware of the necessity to adjust their knowledge according to new requirements imposed by globalisation." (Expert 15).

\section{Knowledge Distance}

A problem faced by the EAAP was that of knowledge distance between partners. Even though it contributes to knowledge flow from advanced to laging organisations, it influences KT effectiveness. If partners have an adequate knowledge base in place, "the transfer of additional knowledge is simplified; if the recipients' knowledge level is too low, they may have difficulty to absorb the knowledge to be transferred directly." (Expert 2). A gap "between partners in terms of their knowledge bases and their different cultural behaviour will influence the success of transferring knowledge" (Expert 16). Additionally, "there is a substantial difference in the understanding of the terms used by the European and the Asian organisations, which seriously affects the cooperation and $K T$ ". (Expert 6). Another pointed out that "a good collaboration needs partners of equal strength; if one partner is too weak (or less developed), collaboration becomes help, which isn't sustainable because it's a one way operation" (Expert 5). Then collaboration tends to rely on the stronger partner, who may have more control and even dominate. To address this, one expert suggested that "an extra-effort is needed from the 
coordinator in explaining the task in a very basic level in order that the partners can understand what they need to do" (Expert 8).

\section{Objectives and Focus}

One important finding was about the need to have clear objectives and focus in KT projects. "It is only possible to transfer knowledge between partners if all partners aim at the same objectives". (Expert 14). Whilst rarely mentioned elsewhere, our experts felt that having clear common objectives and focus should be a priority: "Objectives and methodology should be negotiated among partners." (Expert 17); "Knowledge transfer has to be based on existing needs, requirements and problems of the targeted regions. Thus, a sound background work has to be performed that will provide the necessary elements and success factors for this transnational cooperation”. (Expert 6); also, as one said: “new technology and knowledge transfer need to fit the overall strategy of both parties" (Expert 1).

\section{Transfer Channel}

A variety of KT channels were used by EAAP including training; workshops; networking events; seminars; case studies; surveys; ICT system development; B2B match-making and partnership building activities; business support and technical assistance; institutional support; exchange of personnel, etc.

Over half of the experts agreed that the transfer channel is very important: "the selection of the appropriate communication channels and mechanisms is of vital importance for the success of the KT task" (Expert 6) and the "lead partner's global dissemination channels" (Expert 19) contributed to KT effectiveness. Also, that transfer channels should include: "foreign experts as well as local ones" (Expert 10); and the project should utilise the most appropriate transfer activities and channels; otherwise, "you cannot deepen the partner's competence in a specific field" (Expert 3). 


\section{Selection of Appropriate Partners}

In round two the consensus rate for this factor was 53\%. Partnerships between European and Asian organisations are at the core of the EAAP and the basis upon which the European Commission wishes to promote regional and multilateral networking between the EU and Asia.

"Selection of the appropriate partners in the area addressed by the project ensures effective knowledge transfer and mainly the future implementation of this know-how in the target areas" (Expert 6). This requires the selection of: "European and Asian organisations with previous cooperation experience" (Expert 6). This factor may also shows that the constitution of a partnership is critical in KT projects.

\section{Trust}

Trust is extremely important for knowledge exchange, however, it was last in our list of the ten major factors. Experts pointed out that knowledge transfer: "won't be possible without trust, winwin and good communication" (Expert 9) and that "trust among partners will allow (partners) to overcome problems and unforeseen obstacles" (Expert 17). Some stressed trust's influence on other factors: "trust is the basis of any fruitful collaboration." (Expert 5); "trust is critical for relationship building” (Expert 1), and that it affects KT indirectly through its effect on other factors. It has also been noted [31] that the Chinese are unlikely to share their own 'lessons' with those considered to be 'out-group' - i.e., they are less trusted than those who are considered ingroup.

\section{Openness}

Participants' openness was regarded as an important factor by over half of participants: some stated that: "Openness facilitates positive dialogue" (Expert 11); "openness and acceptance of new methods and differences in cultural approach is important" (Expert 14); and openness "can increases with further acquaintance [among partners]" (Expert 7). Another said that KT is a “...bi-directional knowledge transfer, in a development project, knowledge does not belong to 
only one partner. Even when transferring knowledge (e.g. international best practices), adaptation to local needs is a key condition for success" (Expert 17).

Furthermore, as the EAAP partners were geographically dispersed , an expert commented that "they have experienced more openness in communications among the Asia and European partners by using ICT (Internet and Communication Technology), since the interface in a virtual environment is not personal. The Asian teachers experienced that traditional hierarchical structures were broke [sic] down and communication more easy" (Expert 12).

\section{Other Interesting Factors}

Several other factors were mentioned but did not achieve a high level of agreement, theey included:

Respect - an attitude of mutual regard, admiration or esteem. Six experts pointed out its importance in TKT. "Obviously, when partners work on mutual respect and understanding, KT is enhanced" (Expert 7; "A good project in the development field should (be) based on mutual respect" (Expert 17); "The advance[d] institution will respect under-developed institution, ..." (Expert 9).

Flexibility - This was mentioned by six experts. "The world is moving fast and only projects that can quickly response to the situation are successful" (Expert 5); "Flexibility in budget, schedule etc., are important"'(Expert 5). It can be seen as the ability of an organisation or project to adapt to different circumstances, and is affected by organisational culture, infrastructure, and a mechanism to deal with changing environments.

Policy framework/bureaucratic procedures - This can seriously constrain projects; experts noted that "long bureaucratic procedures limiting the freedom for travelling of some Asian 
people, reduce the opportunity of them to travel to Europe to attend technical visits" (Expert 10);

"there can be policies that restrict knowledge transfer, e.g. lack of IPR (Intellectual Property

Rights) protection in some countries" (Expert 3).

\section{Appendix 2 - Information about Europe Aid Asia sub-programmes:}

- The Asia-Link Programme is an initiative to promote regional and multilateral networking between higher education institutions in Europe and developing countries in Asia. The programme aims to promote the creation of new partnerships and new sustainable links between European and Asian higher education institutions, and to reinforce existing partnerships.

- The Asia IT\&C Programme is to foster economic growth and understanding between Europe and Asia through better awareness, access to, and use of Information and Communication Technologies (ICT). The purpose is to increase direct co-operation between Europe and Asia in the field of ICT; better serve the ICT needs of local communities and citizens, and provide an input into sustainable development.

- The Asia-Pro-Eco Programme is initiated to strengthen the environmental dialogue between Asia and Europe through the exchange of policies, technologies and best practices that promote more resource-efficient, market driven, and sustainable solutions to environmental problems in Asia. The programme aims to support a series of preventive and corrective actions, which materialise in technical solutions that contribute to both quality of life and economic prosperity in Asia.

- The Asia-Invest Programme is primarily intended for potential applicants: intermediaries from the EU and Asia - representatives of business, including chambers of commerce, sector and industry associations, standards and quality institutes and other non-profit multiplier organisations in contact with SMEs and interested in the promotion of trade and investment between the EU and Asia. 